\title{
Sustainable development goals as a challenge for national and global development
}

\author{
Maria Balcerowicz-Szkutnik ${ }^{1}$, Weronika Szkutnik ${ }^{2}$, and Włodzimierz Szkutnik ${ }^{3}$ \\ ${ }^{1}$ University of Economy, Economic College, Katowice, 1-go maja 50, Poland \\ ${ }^{2}$ University of Economy, Economic College, Katowice, 1-go maja 50, Poland \\ ${ }^{3}$ Higher School of Management and Administration, Opole, Niedziałkowskiego 18 - Poland
}

\begin{abstract}
In the article, considerations are based on the implementation of two main goals of the sustainable development strategy. The differences observed in practice in the group of countries with low and medium-low incomes in closing the poverty gap measured by income and the number of people remaining in poverty over a relatively long period can be modeled by measuring the routine intensity of tasks in a given profession. This is indirectly related to an important classical factor in the measurement of knowledge capital and may be important in the study of the relationship between the convergence strength of the analyzed group of countries and the intensity of routine work, which are always negatively correlated with each other.
\end{abstract}

\section{Introduction}

Inequality and poverty are a major problem in the programs of the countries responsible for changes in this aspect. In order to effectively eliminate, for example, poverty in a significant part of Africa in the context of sustainable development (Sustainable Development Goals (SDGs)), it is necessary that by 2030, which was adopted as a border, visible progress is made in involving countries responsible for their willpower in sustainable development policy and results orientation. Based on a lot of research, especially regarding the relatively transparent development of the "action plan for people, planet and prosperity" - developed by the United Nations Institute for Economic and Social Research on Innovation and Technology in Maastricht, you can get a picture of the situation in selected countries, e.g. Africa and Asia. They focus on various aspects of the new monitoring tool designed to measure the impact of the SDG and thus increase the responsibility of governments. This is not 
optimistic, but it allows us to indicate the scale of the problem and the effects of the program implemented under SGD.

\subsection{Sustainable development goals (SDGs)}

The Sustainable Development Goals (SDGs) are 17 global goals set by the UN in 2015 to be met by 2030. Sustainable development goals are part of UN General Assembly resolution 70/1, Agenda 2030. The main two basic goals of sustainable development are:

1. Without hunger

2. Zero poverty

Others relate to psychological aspects (e.g. wellbeing), quality of education, availability of drinking water, clean energy, working conditions and economic growth, industry, innovation and infrastructure, reduction of inequalities, sustainable development of the city and community, responsible consumption and production, climate change, life under water and on land as well as peace and partner purposes. These goals are broad and interdependent. Each of the 17 sustainable development goals has a list of goals measured using appropriate indicators. Sharing data on 17 goals is the key to SDG's success. There are many different tools to track and visualize progress in achieving goals. In his 2019 research article, Anthropologist Jason Hickel [19] questioned the assumption that improving productivity and further economic growth by 3 percent (Goal 8) can be reconciled with ecological sustainability. J. Hickel's findings suggest that this assumption is empirically invalid and "proposed to abolish the aggregation of global growth requirement and introduce quantified targets for resource use per capita with a significant reduction in the number of high-income countries."

\subsection{Implementation of two basic goals in selected countries}

Sustainable Development Goals (SDGs) on poverty pose a new challenge to national and global development. To achieve the sustainable development goal 1 (extreme poverty), i.e. subsistence for less than USD 1.90 per person per day, it must end by 2030; meanwhile, the number of people living in poverty, according to national definitions, must be reduced by half accordingly. Ensuring efficient resource allocation seems to be a fundamental task in achieving these goals [24].

The basic results carried out by the UNU Maastricht Institute for Economic and Social Research in the field of innovation and technology (UNU-MERIT) point to a certain methodology whose clear diagram explains the differences in overcoming extreme poverty and in identifying existing problems. The Institute's research sought to contribute to this goal and was included in a working document entitled (point) "Accessibility of sustainable development goals: myth or reality?". The adopted methodology provides grounds for estimating resource guaranteeing the achievement of goals.

The proposed methodology suggests identifying three main stages. The first applies estimates the annual investment required to prohibit extreme poverty for all people and thus achieve one of two specific objectives. In the second stage, the resource gap was estimated, achieving the second of the specific objectives of halving 
the number of people living in poverty in line with national definitions. In the third stage, the total resource allocation required to achieve the poverty goals under sustainable development was estimated. This was achieved by adding resource requirements to achieve the first two goals, and then converted to a percentage of gross domestic product (GDP).

The starting step of the study was the selection of the five countries studied: Cambodia, El Salvador, Ethiopia, Indonesia, and Senegal. The basic criteria for their selection were three criteria:

location, classification of the World Bank as a country with low or less than average income and data availability [9]. The study estimated the income gap requirement by first measuring the resource gap to eliminate extreme poverty for all people. The results showed that about $5.723 \%$ and $6.17 \%$ of additional investment is needed in the low-income category to fill the poverty gap. Some results are surprising, indicating that obvious patterns cannot be traced directly. It was found, among others, that despite the fact that the incidence of poverty in Ethiopia is lower than in Senegal, the demand for resources is the highest in five countries due to low GDP. An additional $6.2 \%$ of GDP should be allocated to Ethiopia's implementation of the abovementioned sustainable development goals. Senegal must also make a significant contribution to these goals. An additional $4.2 \%$ of GDP is needed to eliminate extreme poverty, and a further $1.6 \%$ of GDP is needed to bring half the population above the national poverty line. Overall, Senegal must spend $5.7 \%$ of GDP to achieve new poverty goals.

As a result of applying these stages in five selected countries, two from the lowincome group (Ethiopia and Senegal) and three others from the below-average income category (Cambodia, El Salvador and Indonesia), it is found that the resources needed to make up the difference in poverty varies and does not always coincide with the amount of income and varies from country to country. The poverty gap in the USD 1.90 line is the smallest in these three countries with below average income, employment in poverty in the poverty line is diverse and incompatible with income, and the number of the poor is the lowest in the three countries with average income [9]. This raises an important issue related to the need to examine the impact of increasing globalization, in reducing the gaps in possible losses in order to potentially increase profit as a result of this process [9].

In the further part of the article, this aspect of the problem will be broadly characterized [6,17]. Main attention will focus on assessing how differences in the content of purposeful and creative tasks are predicted by four factors: technology, globalization, structural changes and the supply of skills $[23,25]$.

\section{Division of routine and innovative tasks}

A wider perspective in considering the relationship between the implementation of sustainable goals and globalization processes initiating the flow of technology enables the division of tasks in the implementation of technologically modernized work on routine and non-routine tasks that require creativity. There are research examples, which has already been mentioned earlier, that unobservable factors of production, 
such as skills necessary for the performance of work or results such as productivity can only be obtained from surveys or evaluated by a group of experts.

The difficulty that arises here is due to the need to unify cognitive tasks performed while implementing the productivity function as a result of expenditure, such as cognitive abilities occurring in different countries. Research methods related to the global division of tasks focus on the differences in work related to these divisions. For an example introduction to the generally described scheme of activities, we will refer to research known from the literature [22] in which microdata come from large-scale surveys conducted in 42 countries of the world. Its data from the study are from three sources: the OECD Program for the International Assessment of Adult Competence (PIAAC), Employment and Productivity Skills of the World Bank (STEP), conducted in middle and low income countries, and the China Urban Labor Survey (CULS), which includes the study STEP based module. The construction of task activities for employees compliant with $\mathrm{O} * \mathrm{NET}$ (American structure of task classification) and covering countries with low, medium and high income is a priority contribution to this research [14,16]. Such a base was in research [22] A priority for the presented approach to the unification of these tasks. The fundamental determinant in assessing the impact of the type of tasks performed when implementing new technology was to determine how the four basic forces are related - technology, globalization, skills supply and structural changes) with the occurrence of differences in tasks performed in individual countries.

A less problematic methodically way was presented in studies documenting the importance of information and communication technologies (ICT) for the demand for tasks in OECD countries [2,12]. This study explores several possible explanations of bias in assessing broadband Internet access options. This gives some evidence that Broadband Internet complements qualified employees in performing abstract nonprogrammatic tasks and replaces unskilled employees in performing routine tasks. Applications based on international databases are another approach to studying the impact of tasks and their intensity on sustainable development, including economic growth. In this aspect, it is reasonable to define measures for "de-routinization " $[11,21]$, group and classify occupations into non-intensive, low, medium and high intensities $[7,18]$ or to combine manual and cognitive tasks.

\subsection{The importance of basic factors for predicting differences and methodological sketch}

The quantitative assessment of the relative importance of four basic functions: technology, globalization of structural changes and the supply of skills in predicting differences in tasks between countries, also depends on the adopted regression model, including the country level category. These four functions are important in predicting differences in the global aggregate sample of all employees.

The coefficients from the estimated regression models have an important role here [22], and the gap distribution is estimated on the basis of the average routine intensity of tasks in individual countries in which the contributions related to the four basic forces were used. The divergence between countries is divided in terms of average routine task intensity and allows the comparison of each country with the US $[4,5]$. 


\subsection{Preparation of data for modeled tasks}

Interesting the data used in the newer study come from three comparable studies: the OECD Program for the International Assessment of Adult Competence (PIAAC), the World Bank Skills Program (STEP) (Du \& Park, 2018) and the third study conducted by Aveo at the China Urban Labor Survey (CULS ) conducted by the Institute of Population and Labor Economics of the Chinese Academy of Social Sciences (CASS). To build survey-based task measures according to those based on $\mathrm{O} * \mathrm{NET}$, identified the harmonized survey questions available in both PIAAC and STEP surveys, whose content is similar to the questions used to construct $\mathrm{O} *$ NET-based task measures [3] A synthetic measure of relative routine task intensity (RTI) is created at employee level using the formula:

$$
R T I=\ln (\text { rcog })-\ln 0.5\left({ }^{n r} \text { analytical }+{ }^{n r} \text { personal }\right),
$$

where $r \operatorname{cog}^{n r}$, analytical and ${ }^{n r}$ personal are, respectively, routine cognitive task levels, non-routine cognitive, analytical task levels, and non-routine, personal cognitive task levels.

\subsection{Cause modeling - Methodology}

The basic tool used to study the impact of the intensity of routine tasks on the factors determining the differences between economic growth of different countries are estimated OLS regression pools in the form of a regression model [10,22]:

$$
R T I_{i j s c}=\gamma_{0}+\gamma_{1} \cdot T_{s c}+\gamma_{2} \cdot G_{s c}+\gamma_{3} S_{i j s c}+\delta \cdot s+\varepsilon_{i j s c}
$$

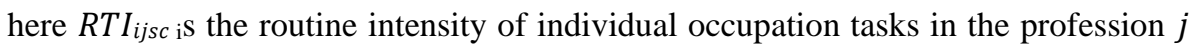
in sector $s$ in country c, Zsc technology used in sector $s$ in country c, Gsc measures globalization in sector $s$ in country c, Eijsc express the individual skills of employees, and $\lambda s$ determined sectoral effects. These regressions are cross-sectional, so they are treated as characterizing assignments of balance for tasks and are not interpreted causally.

Measures in the field of technology, globalization and structural changes in the model (2) are presumed, as measures at the national level, that they are exogenous to the decisions of individual companies and employees. Skills are measured at the individual level, taking into account that education and literacy are mostly predetermined before entering the labour market. There could also analyze skills levels at the sector level and interpret how these changes result without this assumption. To measure for the main technology variable, the percentage of employees in the sector - in the country - who use computers at work is used (PIAAC Literacy Expert Group, 2009).

Regression is estimated for all employees and sub-groups of employees and there is distinguish employees in offshorable and non-off professions. The classification of binder professions is used for this purpose. To assess the relative importance of the four basic factors in predicting differences in tasks between countries, the estimated factor is used to calculate a linear forecast of the intensity of routine tasks at national level $R T \underline{\underline{\underline{\underline{\underline{I}}}}}$. 
For example, the aggregation of countries into three groups is presented based on their level of development and we use unweighted average differences in RTI, all variables explaining all contributions. For employees with high skills, a variance is explained that is similar to that for all employees, and the contribution of technology to variance between countries is the largest (26\%) followed by globalization (10\%), structural changes (10\%) and skills (7\%). The explained variance for employees with medium and low skills (25\% and 23\%) is much smaller than for all employees (57\%), and both structural changes and skills constitute a very small variance. In the case of professions requiring medium skills, like all employees, technology is the most diverse (13\%), compared with $8 \%$ of globalization. However, for low-skilled professions, globalization is the most important factor, which accounts for $21 \%$ of the variance between countries, and technology only accounts for 6\%. Model ratings show that the share of structural changes for all employees and employees in lowskilled professions is negative. Model ratings show that the share of structural changes for all employees and employees in low-skilled professions is negative. This is due to the fact that shares in employment in some sectors, e.g. industry is usually the same for all countries. It is also noticeable that the main impact of skills is their impact on the occupational structure.

Some of the research concerns the nature of work clusters in professions, and then it is interesting to examine how many of the documented relationships between routine intensity and the four basic forces are explained by differences in occupational structure and how much of the differences in occupational tasks result. To answer this question, the specification (of the specification of the comparative task regression test is extended by adding permanent effects of the profession, $\tau o$ :

$$
R T I_{i j s c}=\gamma_{0}+\gamma_{1} \cdot T_{s c}+\gamma_{2} \cdot G_{s c}+\gamma_{3} S_{i j s c}+\tau_{0}+\varepsilon_{i j s c}
$$

This specification enables these variable coefficients of the four main factors to capture their impact among employees of the same profession. Then it is possible to compare the coefficients of this specification with the coefficients estimated using the base specification without determined professional effects. This gives the possibility to deduce to what extent the relationship between these four factors is captured by their impact on the occupational structure and how many relationships exist between occupations. The ratios of professional mannequins are intuitive: employees in highly skilled jobs (ISCO1-3) perform less routine tasks than office workers (ISCO4), while employees in low skilled jobs (ISCO7-9) and sales and service employees (ISCO 5) ) usually perform more routine tasks. However, four basic factors still strongly predict differences in routine intensity even after controlling the profession. Although the absolute values of the coefficients relating to education, reading skills and computer use are slightly lower than in the comparative test specification, none of them loses statistical significance [8,22]. It is known that the coefficients associated with the globalization variable change little and remain statistically significant. 


\section{Conclusion}

The research results of different approaches indicated in literature studies over the impact of globalization on the achievement of SDG's allow noticing the methodological complexity of this issue. Important for presented here in the approach modelling the impact of the intensity of routine tasks on the factors determining the differences between economic growth of different countries is an innovative set of data known from the literature. It allows measuring the content of tasks routine and non-routine of the level of individual employees for many countries at various stages of economic development. Main attention focuses on the new survey-based measures have been approved for compliance with the measures of content of tasks based on the American O*NET network, which have been widely used in the literature existing over many years. In this sketchy approach to these issues, the importance and need for quantifying the country-specific content of the task at work and identifying differences between the content of professional tasks in countries at different stages of development are emphasized. This paves the way for more comprehensive research on the distribution of tasks around the world, which may take into account the variation in task demand across occupations and between countries. The subject matter discussed in the article is interesting because it's model approach explains not only the relationships between factors determining the impact of globalization on the effects achieved in the studied country. It also explains the differences in effects achieved by different countries with a similar impact of the same factors.

\section{References}

1. D. Acemoglu, D. H. Autor, Skills, Tasksand Technologies: Implications for Employment and Earnings. In: Card,D. and Ashenfelter, O. (eds). Handbook of Labor Economics (Amsterdam Elsevier, 2011)

2. A. Akerman, I. Gaarder, M. Mogstad, The Skill Complementarity of Broadband Internet. The Quarterly Journal of Economics, 130, 1781-1824 (2015)

3. D. Autor, M. Handel, Putting Tasks to the Test: Human Capital, Job Tasks, and Wages. Journal of Labor Economics,31, 59-96 (2013)

4. D. H. Autor, F. Levy, R. Murnane, The Skill Content of Recent Technological Change: An Empirical Exploration. Quarterly Journal of Economics 118, 4. (2003)

5. P. G. Bain, P. Kroonenberg, L. O. Johansson et al., Public views of the Sustainable Development Goals across countries Nature Sustainability, 2, 819825 (2019)

6. Z. L. Bárány, C. Siegel, Job Polarization and Structural Change. American Economic Journal: Macroeconomics, 10, 57-89 (2018)

7. M. Biggeri, D. A. Clark, A. Ferrannini et al., Tracking the SDGs in an 'integrated' manner: A proposal for a new index to capture synergies and trade-offs between and within goals. World Development, 122 (2019) 
8. A. Chaudhary, T. M. Brooks, National Consumption and Global Trade Impacts on Biodiversity , 121, 178-187 (2019)

9. P. Chongcharoentanawat, K. Haile, D. Kleine, K. T. Bart, V. Osei Kwadwo, The affordability of the Sustainable Development Goals: MERIT working Papers 027, United Nations University - Maastricht Economic and Social Research Institute on Innovation and Technology (MERIT). Handle: RePEc:unm:unumer:2016027. (2016)

10. J. H. P. Dawes, Are the Sustainable Development Goals self-consistent and mutually achievable? Sustainable Development (2019)

11. S. De la Rica, L. Gortazar, Differences In Job De-Routinization In OECD Countries: Evidence from PIAAC, IZA Discussion Paper No. 9736. (2016)

12. D. Deming, The Growing Importance of Social Skills in the Labor Market. Quarterly Journal of Economics, 132, 1593-1640 (2017)

13. Y. Du, A. Park, Changing Demand for Tasks and Skills in China, Background Report for the World Bank Group (WBG)-Development Research Center under the State Council (DRC) report on New Drivers of Growth in China. (2018)

14. A. Evseev, T. Krasovskaya, V. Tikunov, Planning Sustainable Economic Development, Russian Arctic ISPRS International Journal of Geo-Information, 8 (2019)

15. H. S. Friedman, Causal inference and the Millennium Development Goals (MDG): Assessing whether there was an acceleration in MDG development indicators follong the MDG declaration. Munich Personal RePEC Archive. (2013)

16. C. B. Frey, M. A. Osborne, The future of employment: How susceptible are jobs to computerisation? Technological Forecasting and Social Change, 114, 254-280 (2017)

17. S. Gammage, N. Stevanovic, Gender, migration and care deficits: what role for the sustainable development goals? Journal of Ethnic and Migration Studies, 45, 14 (2019)

18. S. M. Griffiths, The Sustainable Development Goals: an agenda for us all Perspectives in Public Health, 139, 224-225 (2019)

19. J. Hickel, The true extent of global poverty and hunger:Questioning the good news narrative of the Millennium Development Goals, Third World Quarterly (2016)

20. J. Holliday, J. Hennebry, S. Gammage, Achieving the sustainable development goals: surfacing the role for a gender analytic of migration. Journal of Ethnic and Migration Studies, 45, 14 (2019)

21. S. Lee, Role of social and solidarity economy in localizing the sustainable development goals International Journal of Sustainable Development and World Ecology (2019)

22. P. Lewandowski, R. Keister, W. Hardy, S. Górka, Routine and Ageing? The Intergenerational Dividein the Deroutinisation of Jobs in Europe. IBS Working Paper, 01/2017 (2017) 
23. I. Oncioiu, From summits to solutions. Innovations in implementing the sustainable development goals Regional Science Policy and Practice (2019)

24. V. W. Y. Hao, Adaptive reuse in sustainable development International Journal of Construction Management, 19 (2019)

25. Z. Wang, S. Wei, X. Yu, K. Zhu, Measures of Participation in Global Value Chains and Global Business Cycles. NBER Working Paper, 23222, National Bureau of Economic Research, Inc. (2017) 